\title{
X-Ray Diffraction Microscopy of Magnetic Structures
}

\author{
Joshua J. Turner, ${ }^{1,2, *}$ Xiaojing Huang, ${ }^{3}$ Oleg Krupin, ${ }^{4,1}$ Keoki A. Seu, ${ }^{5}$ Daniel Parks, ${ }^{6}$ Stephen Kevan, ${ }^{6}$ \\ Enju Lima, ${ }^{7}$ Kim Kisslinger, ${ }^{7}$ Ian McNulty, ${ }^{8}$ Richard Gambino, ${ }^{9}$ Stephane Mangin, ${ }^{10}$ \\ Sujoy Roy, ${ }^{5}$ and Peter Fischer ${ }^{5}$ \\ ${ }^{1}$ Linac Coherent Light Source, SLAC National Laboratory, Menlo Park, California, USA \\ ${ }^{2}$ Department of Physics \& Astronomy, Stony Brook University, Stony Brook, New York, USA \\ ${ }^{3}$ London Centre for Nanotechnology, University College London, United Kingdom \\ ${ }^{4}$ European XFEL GmbH, Hamburg, Germany 22761 \\ ${ }^{5}$ Lawrence Berkeley National Lab, Berkeley, California 94720, USA \\ ${ }^{6}$ University of Oregon, Eugene, Oregon 97403, USA \\ ${ }^{7}$ Brookhaven National Lab, Upton, New York 11973, USA \\ ${ }^{8}$ Argonne National Lab, Argonne, Illinois 60439, USA \\ ${ }^{9}$ Material Science and Engineering, Stony Brook University, Stony Brook, New York 11794, USA \\ ${ }^{10}$ Institut Jean Lamour, CNRS/Nancy-Universite, Nancy, France
}

(Received 22 March 2011; revised manuscript received 21 May 2011; published 14 July 2011)

\begin{abstract}
We report the first proof-of-principle experiment of iterative phase retrieval from magnetic x-ray diffraction. By using the resonant $\mathrm{x}$-ray excitation process and coherent $\mathrm{x}$-ray scattering, we show that linearly polarized soft $\mathrm{x}$ rays can be used to image both the amplitude and the phase of magnetic domain structures. We recovered the magnetic structure of an amorphous terbium-cobalt thin film with a spatial resolution of about $75 \mathrm{~nm}$ at the $\mathrm{Co} L_{3}$ edge at $778 \mathrm{eV}$. In comparison with soft x-ray microscopy images recorded with Fresnel zone plate optics at better than $25 \mathrm{~nm}$ spatial resolution, we find qualitative agreement in the observed magnetic structure.
\end{abstract}

DOI: 10.1103/PhysRevLett.107.033904

Imaging nanoscale magnetic structures and their ultrafast dynamics is of both scientific and technological importance. Many powerful techniques are available today, ranging from spin polarized STM providing a nearly atomic spatial resolution [1] to ultrafast optical Kerr microscopies providing temporal resolution down to the femtosecond regime [2]. Techniques using the unique properties of polarized soft $\mathrm{x}$ rays, such as photoemission electron microscopy [3] or zone plate based microscopes, are among the most promising candidates for combining spatial and temporal resolution down to fundamental magnetic limits [4].

For instance, spectroholography [5] uses interference with a reference wave instead of focusing optics and is useful for ultrafast imaging [6] at free-electron laser facilities. However, progress has been hampered by the challenge of creating a small enough reference structure [7], which has prevented this technique from achieving wavelength limited spatial resolution. Furthermore, since magnetic holography, as currently implemented, uses the interference between the charge and magnetic scattering channels, circular polarization is required (unless a magnetic reference wave can be used [8]).

Published by the American Physical Society under the terms of the Creative Commons Attribution 3.0 License. Further distribution of this work must maintain attribution to the author(s) and the published article's title, journal citation, and DOI.
PACS numbers: 42.25.Fx, 42.30.Ms, 42.30.Rx, 75.60.Ch

An alternate form of lensless imaging called x-ray diffraction microscopy (XDM) [9] has been developed which offers the possibility to reach the true diffraction limit. Through the use of iterative, mathematical algorithms, the phase of the diffracted x-ray wave field can be reconstructed along with the diffracting structure in real space [10]. This technique has been predominantly used in biological imaging [11], where the simple approximation is typically used between the Fourier space intensity and the real-space charge density. Magnetism, however, cannot be simplified in this way, where the total scattering amplitude must be used: a power series involving charge, electron spin, and x-ray polarization [12]. In certain cases, usually measured at the resonance condition [13], the polarization and spin degree of freedom of the electrons can be such that the x-ray diffraction is due purely to the spin of the electrons of the atomic sites, and not the charge distribution [14].

In this Letter, we demonstrate that the combination of the resonant magnetic scattering process and coherent $\mathrm{x}$-ray diffraction can be used to uniquely unravel the directionality of magnetic domains through imaging via pure phase retrieval. We measure magnetic soft $\mathrm{x}$-ray diffraction by tuning to the $L_{3}$ absorption edge of cobalt in a terbium-cobalt ( $\mathrm{TbCo}$ ) alloy film. The signal arises from a $2 p$ to $3 d$ dipole transition, and probes the magnetic structure of the $d$ electrons. This is applicable to singleshot imaging, and can be performed with linear polarization, something that has not been achieved until now. 
Samples of amorphous TbCo were sputtered on 100-nmthick $\mathrm{Si}_{3} \mathrm{~N}_{4}$ membranes in the ratio of $90 \% \mathrm{Co}$ and $10 \% \mathrm{~Tb}$ with $\mathrm{Ta}(5 \mathrm{~nm}) / \mathrm{Ru}(5 \mathrm{~nm}) / \mathrm{TbCo}(80 \mathrm{~nm}) / \mathrm{Pd}(5 \mathrm{~nm})$ structure. These systems are important for the study of the interplay between delocalized $3 d$ transition metal electrons and localized rare-earth electrons. The in-plane and out-of-plane magnetic hysteresis curves were measured by SQUID magnetometry and are shown in Figs. 1(a) and 1(b), exhibiting strong perpendicular magnetic anisotropy.

A $5 \mu \mathrm{m}$ pinhole of $1 \mu \mathrm{m}$ thick Au was fabricated using a focused ion beam at the Center for Functional Nanomaterials (CFN) and attached to the sample membrane within $130 \pm 10$ microns of the sample using a carbon adhesive. This effectively created an equivalent diameter beam size, and was matched to the transverse coherence length of the beam. The experiment was in transmission at beamline 12.0.2.2 at the Advanced Light Source (ALS), where the sample plane was orthogonal to the beam direction. Measurements were taken using linearly, $\sigma$-polarized x-rays at $778 \mathrm{eV}$, where the bandwidth was $0.2 \%$. This gives a longitudinal coherence length of $800 \mathrm{~nm}$. The sample is placed in UHV at $10^{-9}$ torr with a electromagnet placed outside a nonmagnetic chamber [15]. The diffuse scattering from the domain structure was recorded on a charge coupled device (CCD) at a distance $632.5 \mathrm{~mm}$ from the sample. The CCD has $2048 \times 2048$ pixels with a pitch of $13.5 \mu \mathrm{m}$.

All coherent diffraction patterns used for reconstruction were assembled from a series of multiple exposures taken with different direct beam blocker positions to increase the effective dynamic range of the detector [16]. The longest
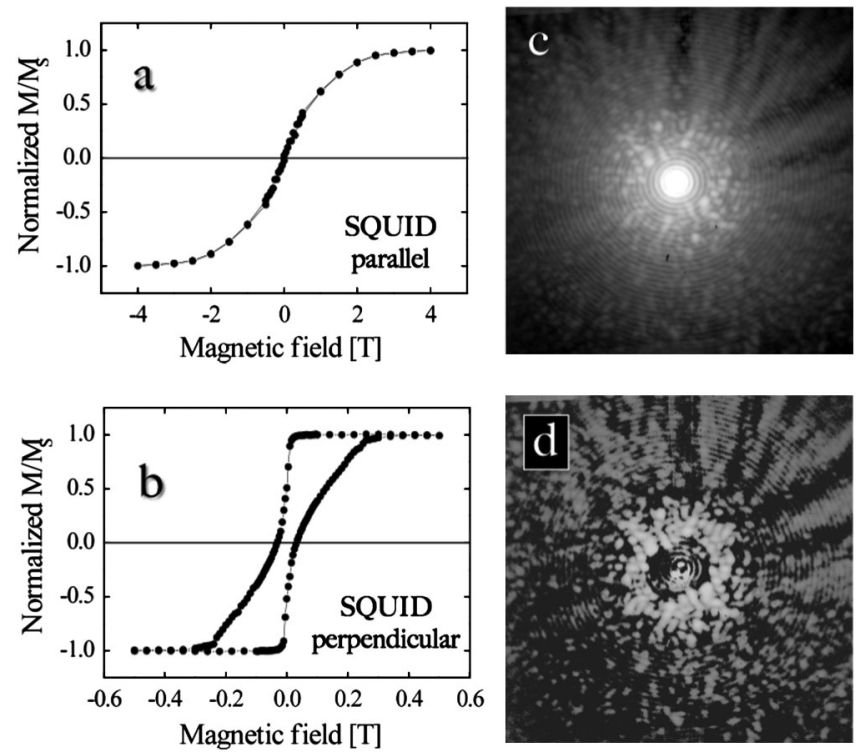

FIG. 1. (a) The magnetization parallel to the sample plane for TbCo using SQUID magnetometry and (b) perpendicular to the film. (c) The speckle pattern of the total scattering: magnetic plus charge. (d) Pure magnetic speckle which is the difference of the coercive and saturated coherent diffraction patterns. acquisition time was $0.2 \mathrm{~s}$ per exposure for the beam blocker centered at $q=0$. This equals about 2000 photons for the highest signal pixels, per acquisition. A set of 100 images was summed to maximize the signal-to-noise ratio. A magnetic field of $0.375 \mathrm{~T}$ was applied in situ perpendicular to the film, completely saturating the sample and removing all diffuse, magnetic scattering. The speckle pattern collected at the coercive field of $3.2 \times 10^{-2} \mathrm{~T}$ maximizes speckle, but is a mixture of charge and magnetic scattering [Fig. 1(c)]. Though this can be used for single-shot imaging, the difference between the coercive field and saturated field speckle patterns [Fig. 1(d)] is used for the reconstruction here to allow for a clean separation of charge and magnetic scattering and to increase contrast.

The relevant elastic scattering amplitude $f$ for the electric dipole transition is given by the following $2 \times 2$ matrix equation [13] summed over all illuminated electrons:

$$
f=f_{c}-i f_{m}^{(1)}\left(\epsilon_{f}^{*} \times \epsilon_{i}\right) \cdot \mathbf{s}+f_{m}^{(2)}\left(\epsilon_{f}^{*} \cdot \mathbf{s}\right)\left(\epsilon_{i} \cdot \mathbf{s}\right)
$$

where $\epsilon$ represents either the incoming or final polarization state, $\mathbf{s}$ is the spin of the electron, and the $f$ 's are charge, and frequency-dependent magnetic scattering amplitudes. The third term is negligible here since both scalar products are nearly zero for linear polarization. The second term is proportional to $\mathbf{k} \cdot \mathbf{s}$, where the unit wave vector $\mathbf{k}$ points in the beam direction [17]. With $\sigma$ polarization, the only nonzero matrix element for this term is the $\sigma$-to- $\pi$ scattering channel [17]. There is no interference term between charge and magnetic scattering, and hence no mixture of scattering channels. This is important because the terms contributing to the total intensity simply become additive:

$$
I=f_{c}^{2}+|s|^{2} f_{m}^{2} \text {. }
$$

The saturated speckle pattern measures the total charge scattering $f_{c}^{2}$ plus the specular component of magnetic scattering. The speckle pattern measured at the coercive point contains the additional diffuse scattering from the domain structure at higher $q$ values, which in turn causes a decrease of the magnetic specular component through energy conservation. To isolate $f_{m}$, the two diffraction patterns are subtracted after being scaled using the power spectral density curve to normalize the charge to magnetic Fourier components from this energy difference. Note that this separation of charge and magnetic scattering cannot simply be made by tuning on and off the resonance energy, as this changes the speckle pattern [18] and does not account for the resonant enhancement of the charge scattering [19]. Phase retrieval is performed on this residual diffraction pattern intensity which is proportional to the Fourier transform of the magnetic density [12], as opposed to the charge density for conventional x-ray diffraction.

Reconstructions were performed using the difference map algorithm [20] and the support constraint used was the pinhole. After the 8000th iteration, the final reconstruction was taken by averaging every second iterate for 2000 
additional iterates. The final image was reproducible with individual random starts. Parameters for the difference map were $\beta=1.15, \gamma_{1}=-1 / \beta$, and $\gamma_{2}=1 / \beta$. The global phase is set from the first averaged iterate and then normalized to following phase iterates and finally summed. The oversampling ratio was $\sigma=18$.

The complex-valued reconstruction of the magnetic speckle pattern is shown in Fig. 2(a). The brightness in the real-space image represents the amplitude of the magnitude of the magnetic component which is always positive. The amplitude should be approximately constant over the domain width regardless of spin direction, and zero for domain walls, since the component of the spins perpendicular to the sample plane is negligible. The fluctuations of amplitude seen over a given, single domain in real space, could be a consequence of distortion on the plane wave state or from a nonuniform illumination of the incident wave field. Another source of uncertainty could be charge "noise" from the imperfect normalization procedure between resonant charge and magnetic scattering.

The phase in the image is represented by hue and is shown by the color key in Fig. 2. The only two colors
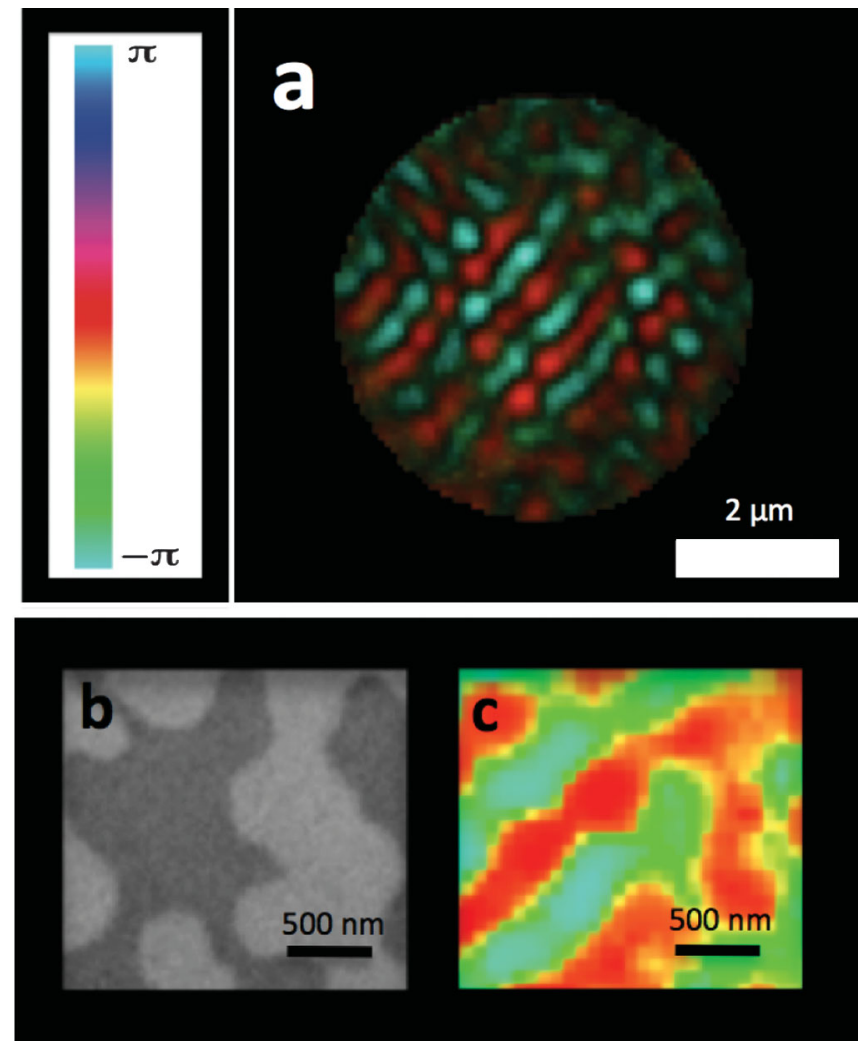

FIG. 2 (color). (a) An image of magnetic domains in TbCo reconstructed by phase retrieval from the magnetic diffuse $\mathrm{x}$-ray scattering. The figure shows the amplitude and phase of the complex image as brightness and color, respectively. (b) A MTXM image of the magnetic domain structure of a different region of the same sample at $22 \mathrm{~nm}$ spatial resolution. (c) A phase-only display of the reconstruction for the same field of view. present in the image differ by $\pi$ in phase, the physical meaning of which is the directionality of the spins over a given domain. This phase sensitivity to spin direction has never been realized experimentally either with XDM or with linear polarization in general. It is recovered by isolating the complex-valued magnetic term in real space, $\left|\rho_{m}\right| e^{i \phi}$, where $\rho_{m}$ is the magnetization density. The phase term is either $e^{i \phi}=-1$ for $\phi=\pi$ or $e^{i \phi}=+1$ for $\phi=0$, up to an arbitrary global phase, and represents the magnetization pointing either up or down. This result is exactly as expected and demonstrates the use of linear light to recover magnetic structure. This has profound consequences as this has only up to now been accomplished with circularly polarized $\mathrm{x}$ rays [21].

To better understand the influence and uncertainty of the pinhole illumination on the sample, we used numerical Fresnel propagation and found that the amplitude fluctuation of the wave front is of order $10 \%$, while a phase variation of $0.04 \pi$ is found. Since we measure a $\pi$-phase shift in the image, the phase component is the much more convincing aspect of the complex image and we use it to compare to a magnetic transmission soft x-ray microscopy (MTXM) image, albeit with higher spatial resolution. We also use the propagator to bring the domain structure into sharper focus, rather than the support, yet only the support is defocused. This is most likely due to the limited resolution currently available, though the overall domain structure is largely unchanged.

Figure 2(b) is a MTXM image recorded at the soft x-ray microscope XM-1 at the ALS [22] with an exposure time of a few seconds and with standard Fresnal zone plate optics providing a $22 \mathrm{~nm}$ spatial resolution. Figure 2(c) shows the phase-only image of the reconstruction. Although the magnetic configuration and sample region were not identical, this comparison shows that there is a good qualitative agreement between the phase in the reconstruction and the x-ray magnetic circular dichroism (XMCD) contrast in the micrograph. In addition, since $\mathrm{XMCD}$ is based on the difference in the absorption of $\mathrm{x}$-ray polarization while the phase contrast in magnetic phase retrieval is strongly dependent on the phase variation of the sample exit wave, this highlights the complementarity of the two techniques.

To further check the reconstruction reliability, we estimate the domain size from micromagnetic theory. The domain size is determined by a balance between the domain wall energy, which increases with domain size, and the demagnetizing field and applied field energy terms [23]. A critical length parameter $l$ is defined as $l=$ $\sigma_{w} /\left(\mu_{0} M_{s}^{2}\right)$ with $\sigma_{w}$ being the magnetic wall energy. For similar samples, this has been measured as $\sigma_{w}=$ $4\left(A K_{u}\right)^{1 / 2}=3.8 \times 10^{-3} \mathrm{~J} / \mathrm{m}^{2}$, where $A$ is the exchange stiffness and $K_{u}$ the anisotropy energy [23]. Using the estimated perpendicular saturation magnetization $M_{s}$ from the hysteresis loop (see Fig. 1) of about $0.2 \mathrm{~T}$ and 


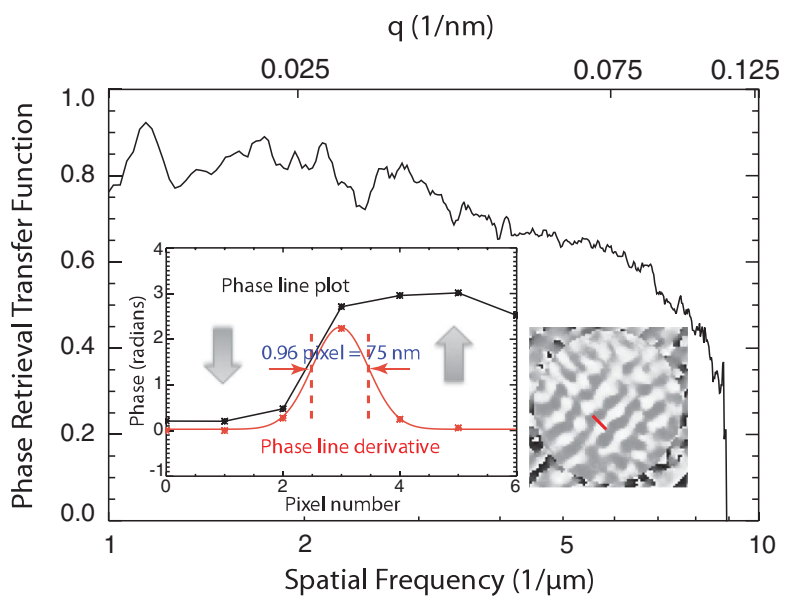

FIG. 3 (color). The Wiener-filtered phase retrieval transfer function [16] of the reconstruction in Fig. 2. This is the ratio of the magnitude of the complex amplitude with the square root of the measured intensity. Inset: a plot across the domains of the phase-only image, along with arrows showing orientation of the domains. The derivative is given by the red curve and gives an estimated resolution of about $75 \mathrm{~nm}$.

the wall energy to calculate $l$, and estimating the critical stable domain size as $8 l$, gives about $100 \mathrm{~nm}$. Therefore the observed domain width is 4 times the critical stable domain size, what is expected for low coercivity. The calculated domain wall width, $\delta_{w}=\pi\left(A / K_{u}\right)^{1 / 2}$ is $5 \mathrm{~nm}$, about $1 \%$ of the width of one domain.

To estimate the spatial resolution from the reconstruction, the Wiener-filtered [16] phase retrieval transfer function (WPRTF) [24] is used and is shown in Fig. 3. This function is the accepted method in XDM to estimate resolution and is a $q$-resolved measure of how well the phases are retrieved. The WPRTF confirms the fidelity of the image and gives a spatial resolution of $7 \mu \mathrm{m}^{-1}$ spatial frequency, or a resolution of $71 \mathrm{~nm}$ [16]. The inset shows the phase-only image along with a curve and its derivative to estimate the resolution. The FWHM of the derivative gives $75 \mathrm{~nm}$, though this is less accurate since it is based on a local region of the image. In the present case, the limited spatial resolution was chamber related, due to the minimum propagation distance between sample and detector being about $0.6 \mathrm{~m}$.

In conclusion, we have used x-ray diffraction microscopy to recover magnetic domain structure by performing phase retrieval on the coherent magnetic speckle pattern of an amorphous $\mathrm{TbCo}$ film. This was demonstrated using linearly polarized light and without the use of a reference wave, such as is used in holography [25], or a permanent mounting of sample to optic, such that a large field of view can in principle be imaged as well [26]. The results are applicable to any coherent $\mathrm{x}$-ray source and the basic method will also work with circularly polarized $\mathrm{x}$ rays. Because of the fact that this experiment uses a support constraint instead of overlapping illumination functions such as in ptychography [27], magnetic phase retrieval is also directly applicable to single-shot imaging at freeelectron laser sources to image spin dynamics on ultrafast time scales. In addition, applying high resolution XDM inside domain walls would furthermore allow the density of pinning sites to be obtained, opening up new avenues to test theories of wall motion coercivity, particularly relevant in exchange bias and multiferroic systems.

We gratefully acknowledge discussions with D. Robins, M. Curtis, A. Scherz, J. Lüning, S. Marchesini, A. Mancuso, and C. Jacobsen. All research in this work was supported by the U.S. Department of Energy, Office of Basic Energy Sciences: partly under Contract No. DEFG02-07ER46128; the ALS, under Contract No. DEAC02-05CH11231; the CFN, under Contract No. DEAC02-98CH10886; and the APS under Contract No. DEAC02-06CH11357.

*joshuat@slac.stanford.edu

[1] A. Wachowiak et al., Science 298, 577 (2002).

[2] Y. Acremann et al., Science 290, 492 (2000).

[3] B. P. Toner et al., J. Electron Spectrosc. Relat. Phenom. 78, 13 (1996).

[4] W. Chao et al., Opt. Express 17, 17669 (2009).

[5] S. Eisebitt et al., Nature (London) 432, 885 (2004).

[6] A. Scherz et al. (unpublished).

[7] D. Zhu et al., Phys. Rev. Lett. 105, 043901 (2010).

[8] S. Roy et al., Nat. Photon. 5, 243 (2011).

[9] J. Miao et al., Nature (London) 400, 342 (1999).

[10] J. R. Fienup. Opt. Lett. 3, 27 (1978).

[11] J. Miao et al., Proc. Natl. Acad. Sci. U.S.A. 100, 110 (2003); D. Shapiro et al., Proc. Natl. Acad. Sci. U.S.A. 102, 15343 (2005); X. Huang et al., Phys. Rev. Lett. 103, 198101 (2009); J. Nelson et al., Proc. Natl. Acad. Sci. U.S.A. 107, 7235 (2010).

[12] M. Blume, J. Appl. Phys. 57, 3615 (1985).

[13] J. P. Hannon et al., Phys. Rev. Lett. 61, 1245 (1988).

[14] D. Gibbs et al., Rev. Sci. Instrum. 60, 1655 (1989).

[15] K. Chesnel et al., Appl. Phys. A 92, 431 (2008).

[16] J. Steinbrener et al., Opt. Express 18, 18598 (2010).

[17] J. P. Hill and D. McMorrow, Acta Crystallogr. Sect. A 52, 236 (1996).

[18] Laser Speckle and Related Phenomena, edited by J.C. Dainty (Springer-Verlag, Berlin, 1974).

[19] Resonant Anomalous X-ray Scattering, edited by G. Materlik, C. J. Sparks, and K. Fischer (Elsevier Publishing, North-Holland, 1994).

[20] V. Elser, J. Phys. A 36, 2995 (2003).

[21] C. Chang et al., Opt. Lett. 31, 1564 (2006).

[22] P. Fischer et al., Mater. Today, 9, 26 (2006).

[23] D. Mergel, Magneto-Optical Recording Materials (IEEE Press, Piscataway, NJ, 2000), Chap. 6.

[24] H. N. Chapman et al., J. Opt. Soc. Am. A 23, 1179 (2006).

[25] L.-M. Stadler et al., Phys. Rev. Lett. 100, 245503 (2008).

[26] D. Stickler et al., Appl. Phys. Lett. 96, 042501 (2010).

[27] P. Thibault et al., Science 321, 379 (2008). 